\title{
-Original-
}

\section{Subcutaneous Angiolipoma: Magnetic Resonance Imaging Features with Histological Correlation}

\author{
Yasuyuki Kitagawa ${ }^{1}$, Masabumi Miyamoto ${ }^{1}$, Shunsuke Konnoㄹ, Akira Makinoㄹ, \\ Go Maruyama ${ }^{1}$, Shinro Takai ${ }^{2}$ and Naoyuki Higashi ${ }^{3}$ \\ ${ }^{1}$ Department of Orthopaedic Surgery, Nippon Medical School Tama Nagayama Hospital \\ ${ }^{2}$ Department of Orthopaedic Surgery, Nippon Medical School \\ ${ }^{3}$ Department of Dermatology, Nippon Medical School Tama Nagayama Hospital
}

\begin{abstract}
Purpose: Despite producing pain, angiolipoma is sometimes misdiagnosed as an ordinary small lipoma, which is usually not associated with pain. Few reports have described magnetic resonance (MR) imaging findings of angiolipoma. The aim of the present study was to clarify the MR imaging features of angiolipoma.

Materials and Methods: The MR imaging findings of 11 lesions in 7 patients were reviewed and compared with histopathological findings.

Results: The MR imaging features of these lesions were the presence of fat nodules with or without areas of low signal intensity on T1- and T2-weighted images. The location of the low-signal-intensity areas varied. The low-signal-intensity areas were mainly in the peripheral portion of 3 lesions and in the central portion of 5 lesions. No or few low-signal-intensity areas were observed in 3 lesions. In the lesions with peripheral low-signal-intensity areas, lesion marginations were well defined, and the lesions were easily recognized as mass lesions. In the lesions with only central low-signal-intensity areas, marginations were poorly defined, and the lesions were not easy to recognize as mass lesions. In the lesions with few or without lowsignal-intensity areas, marginations were invisible. Histopathological studies indicated that the low-signal-intensity areas on $\mathrm{T} 1$ - and $\mathrm{T} 2$-weighted images corresponded to areas of dense capillary proliferation. In lesions with few or without low-signal-intensity areas on MR images, capillaries were thinly spread over almost the entire lesion area.

Conclusion: The MR imaging features of angiolipoma are fat nodules with or without lowsignal-intensity areas of various size and location on T1- and T2-weighted images.

(J Nippon Med Sch 2014; 81: 313-319)
\end{abstract}

Key words: angiolipoma, magnetic resonance imaging, histological correlation, pain, soft tissue

\section{Introduction}

Angiolipoma, originally described in 1960 by
Howard and Helwig ${ }^{1}$, consists of mature adipocytes and branching capillary-sized vessels, which usually contain fibrin thrombi ${ }^{2}$. Angiolipomas occur most often in the forearm, followed by the trunk and

Correspondence to Yasuyuki Kitagawa, MD, Department of Orthopaedic Surgery, Nippon Medical School Tama

Nagayama Hospital, 1-7-1 Nagayama, Tama, Tokyo 206-8512, Japan

E-mail: kitayasu@nms.ac.jp

Journal Website (http://www.nms.ac.jp/jnms/) 
proximal upper extremity, and typically present as multiple small $(<2 \mathrm{~cm}$ in diameter), painful subcutaneous lesions ${ }^{2}$. Spinal angiolipoma and intramuscular hemangioma, which were previously referred to as infiltrating angiolipoma, are distinct clinical entities².

However, despite producing pain, angiolipoma is sometimes misdiagnosed as an ordinary small lipoma, which is usually not associated with pain. Alternatively, angiolipoma may be difficult to differentiate from other small, painful soft-tissue tumors, such as vascular leiomyoma, glomus tumor, schwannoma, or neurofibroma. We believe that MR imaging might be a useful noninvasive method of diagnosing angiolipoma before surgery, as it is for many other soft tissue tumors. However, few reports have described the MR findings of subcutaneous angiolipom $\mathrm{a}^{3-5}$. Because subcutaneous angiolipoma is a small nodule with mild symptoms, MR imaging is not frequently performed. The purpose of the present study was to clarify the MR imaging features of subcutaneous angiolipoma through a review of MR images and a comparison with histopathological findings in our patients.

\section{Materials and Methods}

This study was approved by our institutional review board and was conducted in accordance with the Declaration of Helsinki. We reviewed the MR images of 11 lesions in 7 consecutive patients with angiolipoma, which were treated and histopathologically confirmed in our hospital from July 2008 through June 2012, using medical records, MR images, and histological slides. The patients were 5 men and 2 women with a median age of 40 years (age range, 19-52 years). Two patients had 3 lesions each, and the total number of lesions studied was 11. Six lesions were in the forearm, 4 were in the proximal upper extremity, and 1 was in the lower back.

\section{MR Imaging}

The MR imaging of all lesions was performed with 1.5-T units but at various hospitals. The fields of view were 16 to $35 \mathrm{~cm}$, slice thicknesses were 0.8 to
$8 \mathrm{~mm}$, and slice gaps were 0.3 to $5.5 \mathrm{~cm}$. Matrices of 90-372 × 180-560 were used. Axial and either sagittal or coronal images were obtained for 7 lesions, coronal and sagittal images for 3 lesions, and an axial image only for 1 lesion. Both T1-weighted spin-echo imaging (pulse sequences: 380-914/8-15 [repetition time/echo time]) and T2-weighted spinecho or fast spin-echo imaging (pulse sequences: 2,200-4,000/80-100 [repetition time/echo time]) were performed for all lesions. A fat-suppression technique was applied to 7 lesions, and fatsuppressed postcontrast images were obtained for 2 lesions.

\section{MR Image Analysis}

Two independent readers, who were aware of the diagnosis of angiolipoma, analyzed the MR imaging features. When the readers disagreed, they reached a consensus through discussion. They assessed signal intensity, margination, and enhancement. The signal intensities of the lesions were compared with those of subcutaneous fat. The marginations were classified as well defined, poorly defined, or invisible. Enhancement effects were assessed by comparing postcontrast images and precontrast images. Findings of all MR images, particularly those of MR images in the planes closest to those of representative histological specimens, were studied and compared with histopathological findings. Histopathological slides were available for all lesions.

\section{Results}

Clinical data of the lesions are summarized in the Table. Only 2 lesions were painful. All lesions were subcutaneous lesions, and all were marginally excised. At a mean follow-up period of 13 months (range, 1-47 months), there were no signs of recurrence.

The MR imaging features of these lesions were the presence of fat nodules with or without lowsignal-intensity areas of various amounts on T1- and T2-weighted images (Table). Eight lesions showed fatty nodules with low-signal-intensity areas of various size and location on T1- and T2-weighted MR images. Only very small parts of the low-signal- 
Table Clinical data, MRI findings, and histological findings

\begin{tabular}{|c|c|c|c|c|c|c|c|}
\hline \multirow[b]{2}{*}{$\begin{array}{l}\text { Lesion } \\
\text { number }\end{array}$} & \multirow[b]{2}{*}{$\begin{array}{l}\text { Age } \\
\text { (years)/ } \\
\text { Sex }\end{array}$} & \multirow[b]{2}{*}{ Location } & \multirow[b]{2}{*}{ Pain } & \multirow[b]{2}{*}{$\begin{array}{l}\text { Maximal } \\
\text { diameter } \\
(\mathrm{mm})\end{array}$} & \multicolumn{2}{|l|}{ MRI } & \multirow[b]{2}{*}{$\begin{array}{l}\text { Capillary proliferation on } \\
\text { histologic examination }\end{array}$} \\
\hline & & & & & $\begin{array}{l}\text { Location of } \\
\text { low-signal-intensity } \\
\text { areas in fatty nodule }\end{array}$ & Margination & \\
\hline 1 & $30 /$ male & forearm & - & 14 & peripheral & well defined & dense peripherally \\
\hline $2 \mathrm{a}^{\mathrm{a}}$ & $44 /$ male & upper arm & - & 35 & (no or few) & poorly defined & thinly spread throughout \\
\hline $2 b^{\mathrm{a}}$ & & upper arm & - & 20 & (no or few) & poorly defined & thinly spread throughout \\
\hline $2 c^{a}$ & 44/male & forearm & - & 25 & (no or few) & poorly defined & thinly spread throughout \\
\hline 3 & $19 /$ male & forearm & - & 14 & central & invisible & dense centrally \\
\hline 4 & $52 /$ female & forearm & + & 14 & peripheral & well defined & dense centrally \\
\hline 5 & $34 /$ female & low back & - & 13 & central & poorly defined & dense centrally \\
\hline $6 \mathrm{a}^{\mathrm{b}}$ & 43/male & upper arm & + & 23 & central & poorly defined & dense centrally \\
\hline $6 b^{b}$ & & upper arm & - & 5 & central & poorly defined & dense centrally \\
\hline $6 c^{b}$ & & forearm & - & 10 & central & poorly defined & dense centrally \\
\hline 7 & 40/male & forearm & - & 15 & peripheral & well defined & dense peripherally \\
\hline
\end{tabular}

same patient; bsame patient

intensity areas on T1-weighted images appeared as high-signal-intensity areas on T2-weighted images.

The location of the low-signal-intensity areas varied. The T1-weighted low-signal-intensity areas were located mainly in the peripheral portions of 3 lesions and in the central portion of 5 lesions. The other 3 lesions showed only fatty nodules with few or without low-signal-intensity areas. In lesions with peripheral low-signal-intensity areas, marginations were well defined (Fig. 1). In lesions with only central low-signal-intensity areas, marginations were poorly defined in 2 lesions (Fig. 2) and invisible in 1 lesion; therefore, recognizing these lesions as mass lesions was difficult. The nonfat components showed high-signal-intensity areas on fat-suppressed T2weighted images in 5 of 7 lesions (Fig. 1). These components were enhanced on fat-suppressed T1weighted images after gadolinium administration in 1 of 2 lesions. In lesions with few or without lowsignal-intensity areas, the signal intensities of the lesions were similar to that of fat, and marginations were poorly defined (Fig. 3).

Macroscopically, all lesions were multilobular and yellow or orange. The maximum diameters of excised materials ranged from 5 to $35 \mathrm{~mm}$ with a median of $14 \mathrm{~mm}$. Microscopically, the lesions consisted of mature adipocytes, showed capillary proliferation, and were encapsulated with thin fibrous membranes. The low-signal-intensity areas on T1-weighted images corresponded to areas of dense capillary proliferation (Fig. 1, 2). In the lesions with few or without low-signal-intensity areas on MR images, histopathological examination showed thin spreads of capillaries over most of the lesion area (Fig. 3). No relationship was found between pain and capillary density or distribution. The results of comparisons of MR findings and histopathological findings are summarized in the Table.

\section{Discussion}

To the best of our knowledge, the MR imaging features of subcutaneous angiolipoma have been described in only a few previous case reports ${ }^{3-5}$. In these reports, angiolipomas were described as fatty masses with low-signal-intensity areas on T1weighted images. However, the low-signal-intensity areas on T1-weighted images appeared as highsignal-intensity areas on $\mathrm{T} 2$-weighted images and were highlighted on fat-suppressed images and short-tau inversion recovery (STIR) images. In the present study, the low-signal-intensity areas, which corresponded to areas of dense capillary proliferation, varied in location, and this variation affected the ability of the lesions on MR images to be correlated with histopathological findings.

The present study has shown that marginations of subcutaneous angiolipomas are well defined when low-signal-intensity areas are seen in the peripheral 

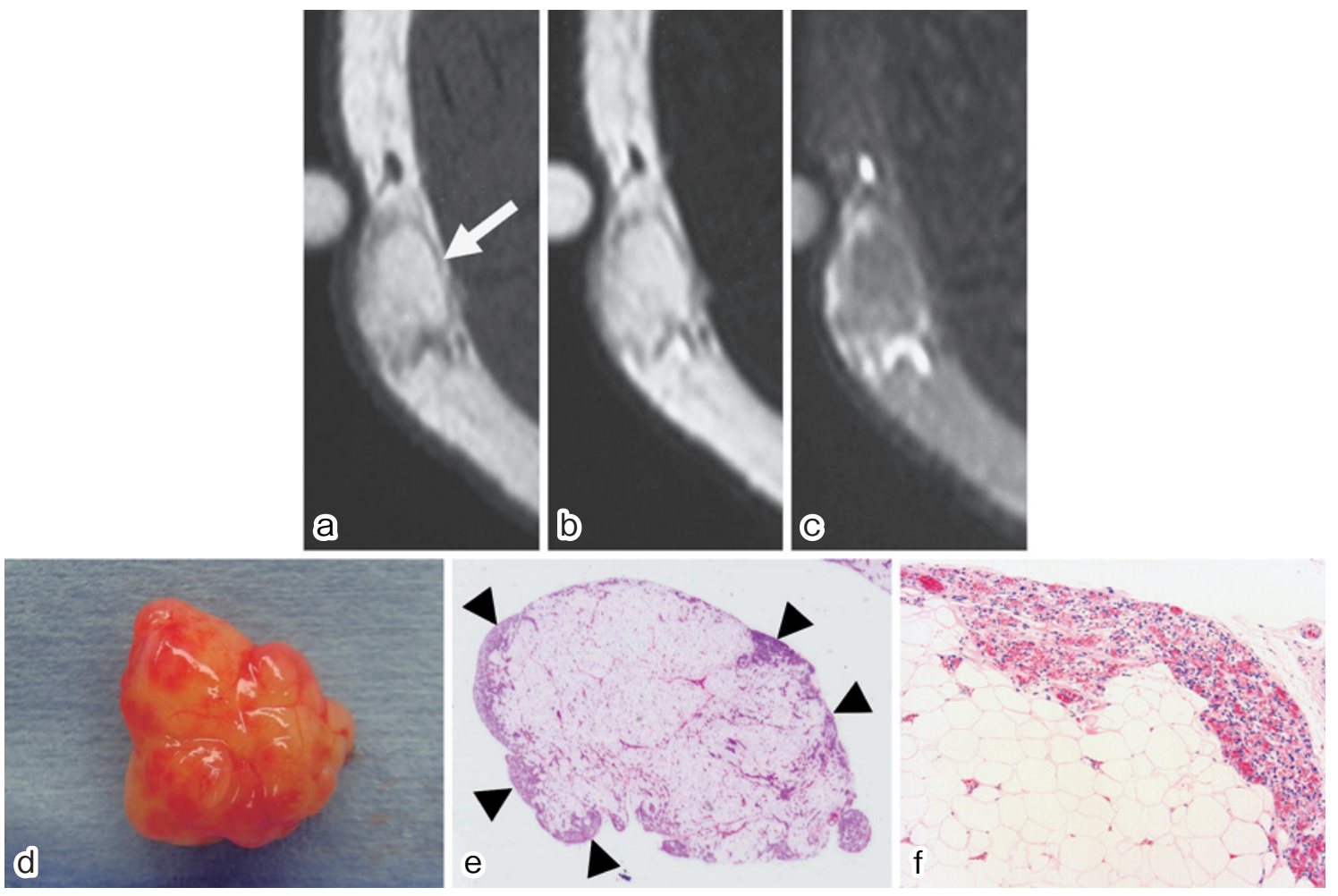

Fig. 1 Angiolipoma of the forearm of 52-year-old women with a tender forearm nodule (lesion 4). a An axial T1-weighted image shows a well-defined fatty nodule (arrow) with multiple small low-signal-intensity areas in the peripheral portion. b A T2-weighted image also demonstrates multiple small low-signalintensity areas, which are slightly smaller than those on the T1-weighted image. $\mathbf{c}$ A fat-suppressed T2-weighted image highlights the low-signal-intensity areas observed on T1- and T2-weighted images, changing the low-signal-intensity areas to high-signal-intensity areas in 5 of 7 lesions. d Gross appearance shows a multilobular surface. e A low-power photomicrograph shows an area of dense capillary proliferation in the peripheral portion (arrowheads) which corresponds to the low-signalintensity areas on MR images (hematoxylin and eosin staining). f A medium-power photomicrograph shows dense capillaries, some of which contain fibrin clots (hematoxylin and eosin staining)

portion on MR images but are poorly defined when such areas are not seen. We speculate that these marginations are obscure in angiolipomas without peripheral low-signal-intensity areas because multiple factors, including thin lesion capsules, similarity of signal intensities of the lesions to those of fat, smallness of lesions, and multilobularity, make the lesions nearly indistinguishable from adjacent fat.

Angiolipomas have varying degrees of capillary proliferation, ranging from a small angiomatous focus to a large area that replaces most of the fatty area; however, peripheral capillary proliferation is the most common finding. Accordingly, we believe that MR findings, which are affected by dense capillary proliferation, may also vary. The MR findings in the present study may be an example of such variation; therefore, further accumulation of MR findings is necessary to more accurately define the MR imaging features of angiolipoma.

The typical clinical manifestations of angiolipoma, multiple small $(<2 \mathrm{~cm}$ in diameter), slightly firm subcutaneous nodules of the forearm and dull pain, usually lead to a correct diagnosis, but the clinical manifestations are not always typical ${ }^{2}$. The differential diagnosis can also include subcutaneous lipoma and painful small subcutaneous tumors, such as glomus tumor, vascular leiomyoma, schwannoma, neurofibroma, and hemangioma. We believe that angiolipomas might be differentiated from such small, painful subcutaneous tumors on the basis of the signal intensity on MR imaging. However, differentiating angiolipoma from lipoma is difficult. Although typical lipomas show the same intensity as 

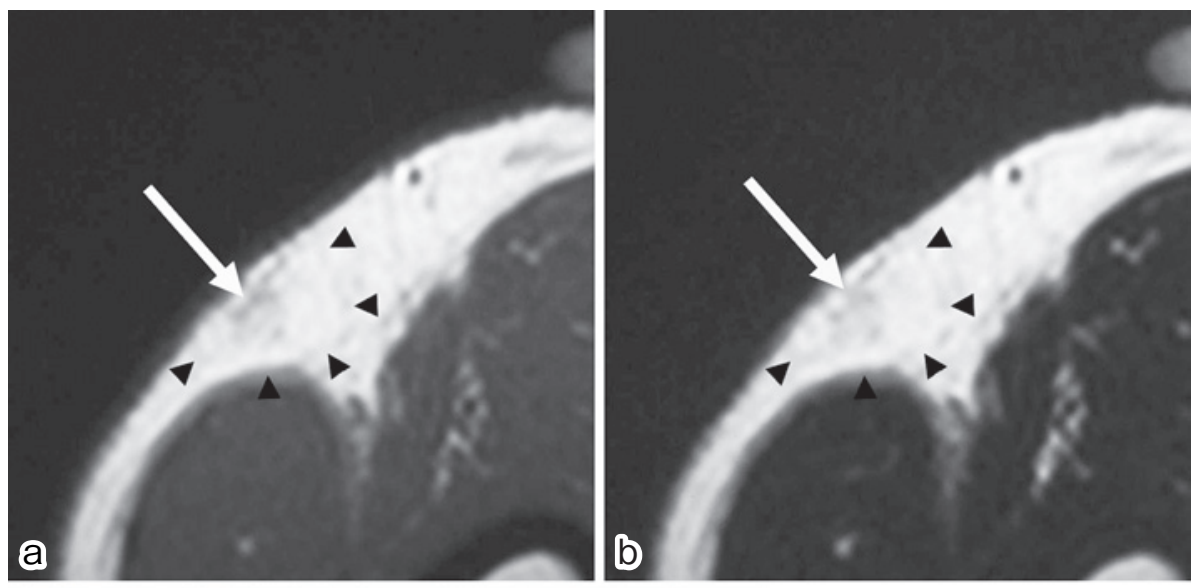

C

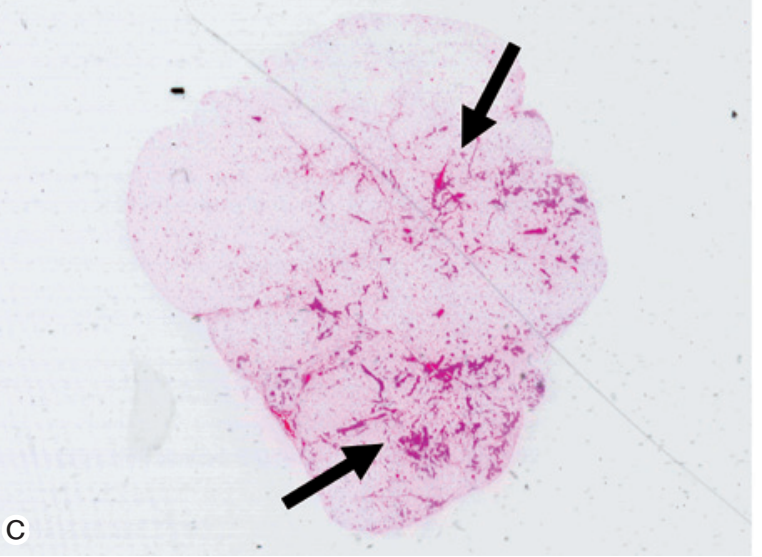

Fig. 2 Angiolipoma of the proximal upper extremity of 43-year-old man with multiple upper-extremity nodules associated with dull pain (Lesion 6b). a An axial T1weighted image shows a poorly defined fatty nodule (arrowheads) with multiple small low-signal-intensity areas (white arrow) in the central portion. b A T2-weighted image demonstrates an poorly defined fatty nodule (arrowheads) with slightly fewer areas of low-signal-intensity (white arrow) than the T1-weighted image. c Low-power photomicrograph shows dense capillary proliferation in the central portion (black arrows), which corresponds to the low-signal-intensity areas on MR images (hematoxylin and eosin staining)

subcutaneous fat, regardless of the pulse sequence on MR images, lipomas sometimes show internal nonfat components that resemble septa or nodules ${ }^{6}$. Galant et al. have reported that hyperintense nodules or septa or both were seen on fatsuppressed T2 images or STIR images of only $11.7 \%$ of lipomas; ; in contrast, nonfat components of 5 of 7 angiolipomas in the present study showed highsignal-intensity areas on fat-suppressed T2-weighted images. Therefore, fat-suppressed T2 images and STIR images might be useful for differentiating angiolipomas from lipomas.

Pain is an important manifestation of angiolipoma, but its cause remains unclear. The relationship of vascularity to pain in angiolipoma is still being investigated ${ }^{12.28}$. As histological features of angiolipoma, vascular channels contain fibrin thrombi, and mast cells are often conspicuouss. However, how these features relate to pain is unclear. When an angiolipoma is not associated with pain, it can be followed up without surgical treatment. Angiolipoma is not thought to undergo malignant transformation.

The present study has several limitations. First, this study was retrospective. Second, the conditions of MR imaging were different for each lesion. Third, the number of lesions was small, particularly for MR imaging with enhancement, which was performed for a limited number of lesions. Fourth, image analysis was performed by nonblinded readers who 

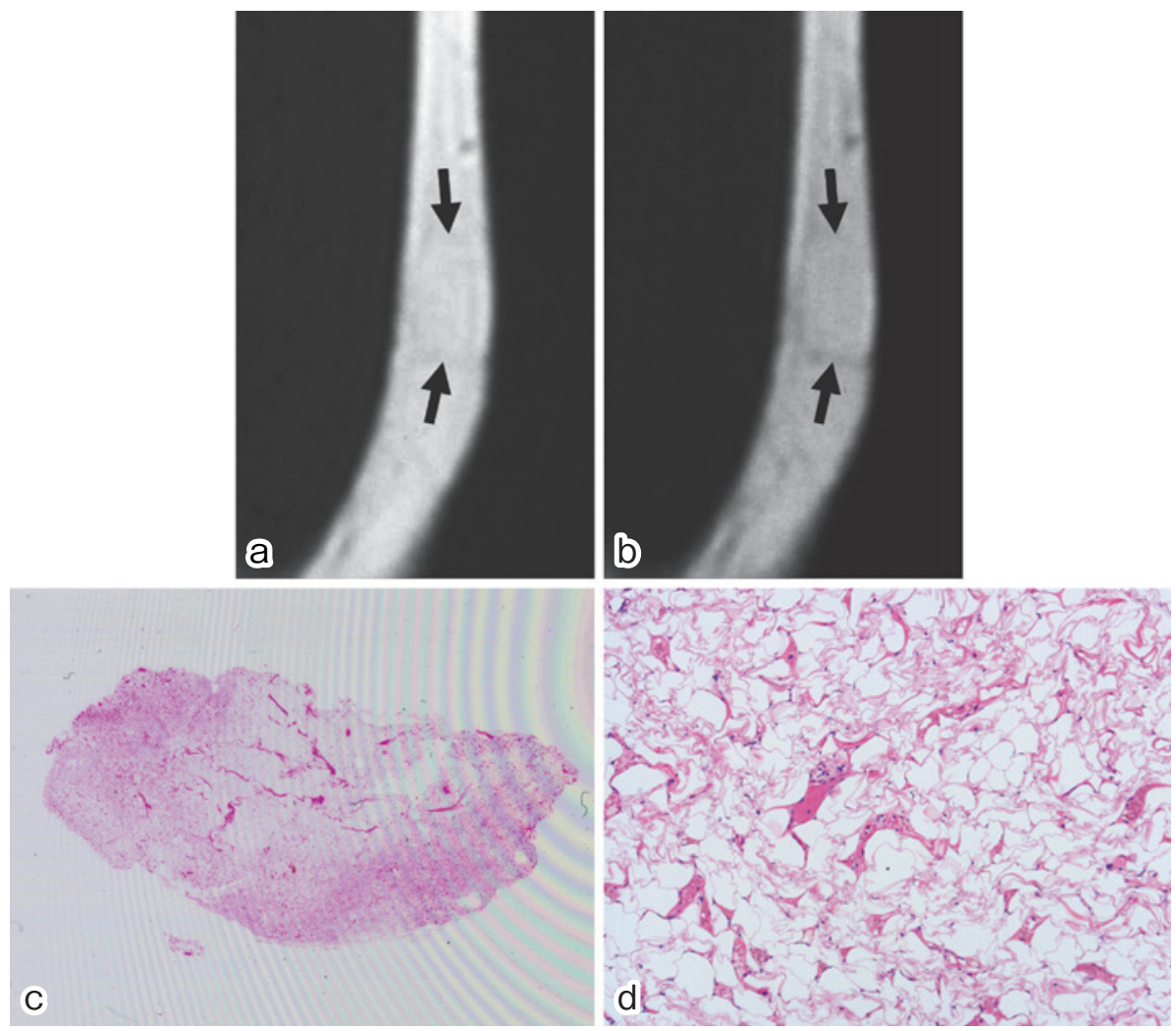

Fig. 3 Angiolipoma of the forearm of 44-year-old man with multiple nontender upperextremity nodules (Lesion 2a). a A coronal T1-weighted image shows a poorly defined fatty nodule (arrows) without low-signal-intensity areas. b A T2weighted image shows similar findings to the T1-weighted image (arrows). c A low-power photomicrograph shows capillaries thinly spread over most of the lesion area (hematoxylin and eosin staining). d A medium-power photomicrograph shows sparse capillary proliferation (hematoxylin and eosin staining).

were aware of the diagnosis of angiolipoma. Fifth, the histopathological comparative studies were performed with histological specimens that did not always have planes identical to those of the MR images.

\section{Conclusions}

The present study has found that angiolipomas are observed on T1- and T2-weighted images as fatty nodules with or without areas of low signal intensity of various size and location which histopathologically correspond to areas of dense capillary proliferation. We believe that awareness of these variations of MR findings, particularly whether these areas are peripheral or central, will be important in diagnosing and evaluating this tumor with MR imaging. The results of the present study suggest that MR imaging is a useful noninvasive preoperative method of diagnosing angiolipoma.

Conflict of Interest: The authors declare that they have no conflict of interest for this study.

\section{References}

1. Howard WR, Helwig EB: Angiolipoma. Arch Dermatol 1960; 82: 924-931.

2. Sciot R, Mandahl N: World Health Organization classification of tumours: tumours of soft tissue and bone (Fletcher CDM, Unni KK, Mertens F, eds), 2002; IARC Press, Lyon.

3. Grivas TB, Savvidou OD, Psarakis SA, et al: Forefoot plantar multilobular noninfiltrating angiolipoma: a case report and review of the literature. World J Surg Oncol 2008; 30: 6-11.

4. Murphey MD, Carroll JF, Flemming DJ, Pope TL, Gannon FH, Kransdorf MJ: From the archives of the AFIP: benign musculoskeletal lipomatous lesions. Radiographics 2004; 24: 1433-1466.

5. Kim JY, Park JM, Lim GY, Chun KA, Park YH, Yoo JY: Atypical benign lipomatous tumors in the soft tissue: radiographic and pathologic correlation. J 


\section{MR Imaging of Angiolipoma}

Comput Assist Tomogr 2002; 26: 1063-1068.

6. Kitagawa Y, Tamai K, Kim Y, Hayashi M, Makino A, Takai S: Lipoma of the finger with bone erosion. J Nippon Med Sch 2012; 79: 307-311.

7. Galant J, Martí-Bonmatí L, Sáez F, Soler R, AlcaláSantaella R, Navarro M: The value of fat-suppressed T2 or STIR sequences in distinguishing lipoma from well-differentiated liposarcoma. Eur Radiol 2003; 13: 337-343.

8. Goldblum JR, Folpe AL, Weiss SW: Benign lipomatous tumors. In Enzinger and Weiss's soft tissue tumors, 6th ed. 2014; pp 443-483, Elsevier, Philadelphia.

(Received, February 21, 2014)

(Accepted, May 8, 2014) 